\title{
Vitamin D - Too Little, or Too Much in the Elderly and the Obese?
}

\author{
István G Télessy* \\ MedBioFit Lpc, Department of Pharmaceutics, University of Pécs, Faculty of Pharmacy, Hungary
}

\begin{abstract}
Vitamin D deficiency became a very popular theme in the medical literature. Thank the global scientific research activity, much is known about vitamin D (VD) however, the sphere of indication of supplementation and the dosage is still a matter in dispute, especially in some groups of patients. A lot of assessment support that more than half of the population is in VD deficit and supplementation is needed. Due to uncontrolled information (internet) and the free availability of VD-containing products, the recommended daily dose is often much less or even more than needed. The risk for hypervitaminosis is existing burden however the symptoms are rarely recognized by the population. This mini-review discuss the main facts and focus to the evidence-based optimal dosage of vitamin D in elevated age and in individuals living with overweight/obesity.
\end{abstract}

KEYWORDS: Vitamin D; Deficiency; Elderly; Obesity; Dosage; Symptoms

\section{INTRODUCTION}

Vitamin D is an essential fat-soluble vitamin. Textbooks give dietary reference intakes according to age, sex, gravidity, lactation, etc. but people are not able to judge their daily VD intake or don't take the figure or the intentions for need of sunlight exposure seriously. Maybe therefore, high prevalence of hypovitaminosis is detected in almost every area of the world [1-5]. Moreover, women, the elderly and obese people are at higher risk for hypovitaminosis than others.

Vitamin D - under physiological conditions - is produced in the skin, however the endogenous production often is not enough, especially nowadays, due to the high air pollution [6]. Thus, most of VD and its precursors are taken up with food. The endogenously produced and/or from exogenous sources absorbed amounts of VD distributes via circulation into the organs and tissues. The serum levels of $<20 \mathrm{ng} / \mathrm{ml}(<50 \mathrm{nmol} / \mathrm{L})$ and $20-30 \mathrm{ng} / \mathrm{ml}$ (50-75 nmol/L) considered as VD deficiency and VD insufficiency, respectively. The low concentrations dispose human beings to comorbidities like cardiovascular, infectious, metabolic, and cognitive disorders.
The supplementation is usually offered by fortified food and dietary supplements or via medical therapy (medicines).

A recent study revealed that by increasing age VD deficiency is steadily increasing [2]. Elderly and obese people are more endangered to hypovitaminosis. Therefore, NIH (USA) recommend an elevated daily dietary allowance (800 IU/D) for individuals above 70 years and for those BMI is $>30 \mathrm{~kg} / \mathrm{m}^{2}$ or obese individuals who have undergone gastric bypass surgery [7]. Here we review the risky conditions and therapeutic options.

\section{Brief Chemical-Biochemical Background}

Vitamin D deficiency as a special form of undernutrition has been discovered ca. 100 years ago. Identification of the first vitamin D goes back to the twenties of last century in the bulk of UV-radiated ergosterol this was a crude mixture called vitamin D1 but later it became clear that this was a mixture of various sterine-type compounds. Further purification led to the crystallized ergocalciferol (vitamin D2). Some years later research with UV-irradiation of the 7-dehydrocholesterol resulted in discovery of cholecalciferol
Quick Response Code:

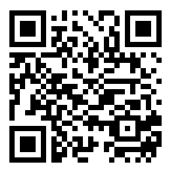

Address for correspondence: IG Telessy PharmD, PhD. MedBioFit Lpc, Department of Pharmaceutics, University of Pécs, Faculty of Pharmacy, Hungary

Received: June 27, 2020 Published: July 14, 2020

How to cite this article: István GT. Vitamin D - Too Little, or Too Much in the Elderly and the Obese?. 2020 - 2(4) OAJBS.ID.000190. DOI: 10.38125/OAJBS.000190 
(vitamin D3). The step-by-step process is demonstrated in Figure 1. The difference of the vitamins D2 and D3 is just the double bond between C22 and C23 and a methyl radical on C24 (Figure 2), and both molecules are transformed enzymatically to 25-OH-D in the liver. Both provitamins are used as dietary supplement. Their names (-calciferol) refer to their role in Ca++-transport. The basic compound ergosterol contains one hydroxyl-group in the position 3 of the sterane-skeleton. During the gradual metabolism (Figure 3 ) first in the liver, one more hydroxyl-group addition to the structure in position 25 (25-OH-cholecalciferol), and later on one more in the kidney in the position 1 resulting 1,25-OH2-cholecalcicerol. Therefore 25-hydroxy-cholecalciferol often named as calcidiol and 1,25-dihydroxy-cholecalciferol named as calcitriol (abbreviated 1,25-DHCC). The physiological vitamin D effect is attributed to the final (active) metabolite calcitriol however the vitamin D name is used to one oor mixture of metabolites VD2 and VD3 (correctly "vitamins D"). Vitamin D dose is usually given in International Units (IU), 1 IU = 25 ng cholecalciferol-equivalent compounds.

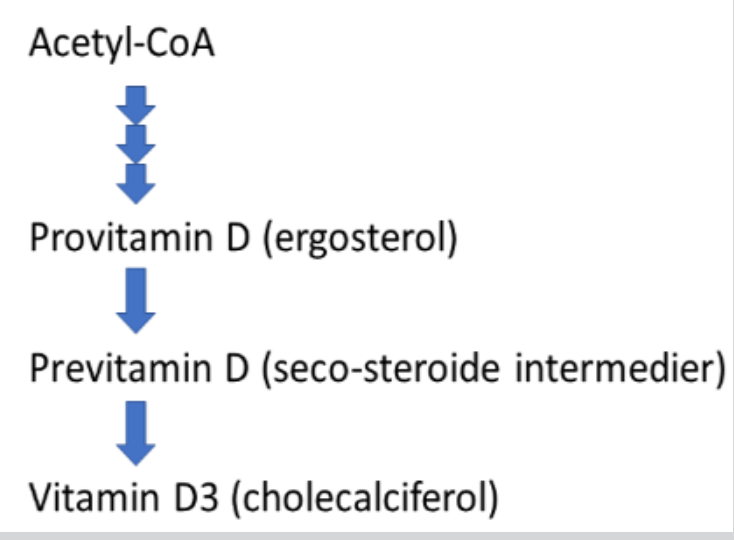

Figure 1: Endogenous formation of vitamin D3.

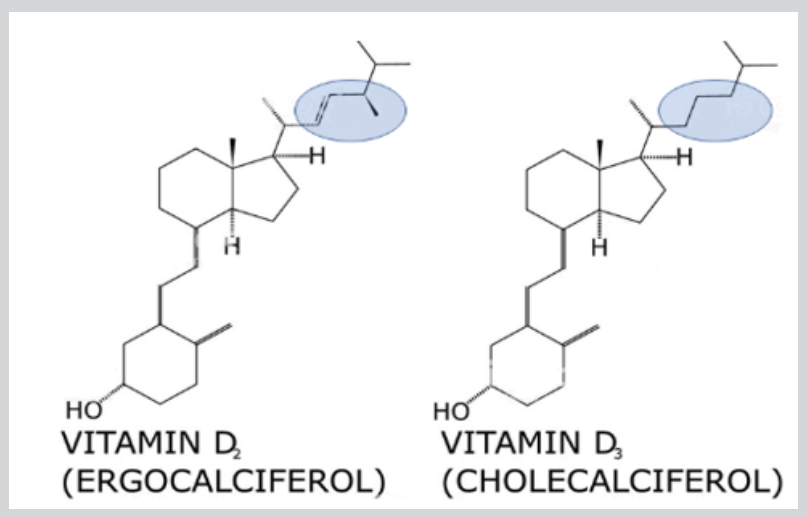

Figure 2: The difference between vitamin D2 and vitamin D3.

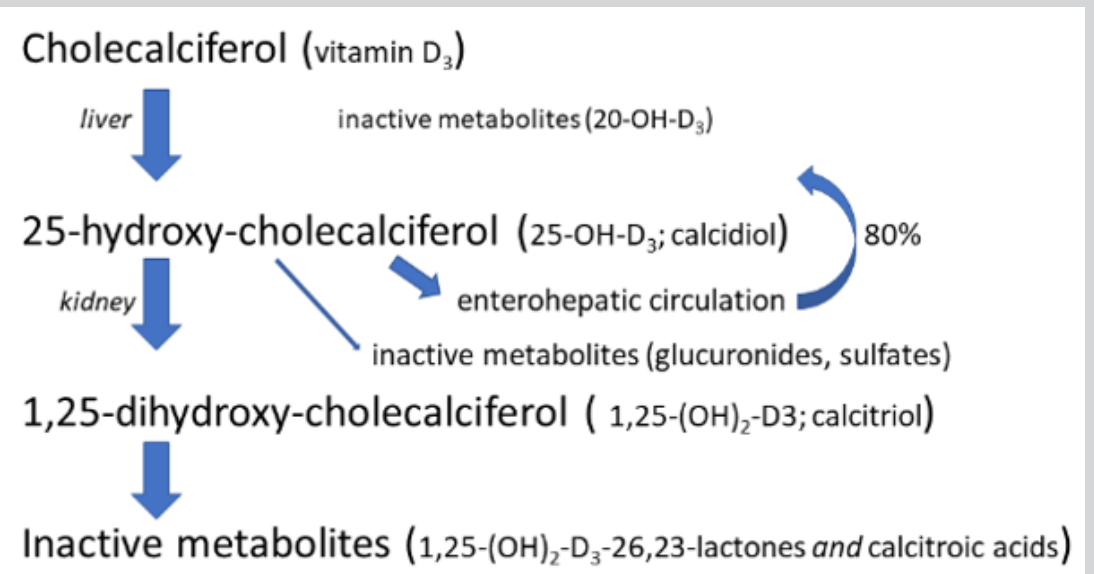

Figure 3: The fate of vitamin D3.

In case of VD supplementation magnesium-supplementation can be dangerous because of risk of hypermagnesemia viz. vitamin D increase the intestinal absorption of magnesium as well.

\section{Brief Physiological Background}

Endogenous production of vitamin D3 originates from acetyl-CoA and is manufactured via multiple steps before reach skin where after UV-B exposure cholecalciferol is born [8]. Although
VD is not synthesized by and released from an endocrine gland the vitamin D, as steroid-originated compound, has hormone-like mechanism of action, pathway and functions similar to many other steroids. First, its classical role in the calcium homeostasis and bone-development should be mentioned. The calcium homeostasis is basically regulated by three "calciotropic" hormones: the parathyroid hormone (PTH), the calcitonin and the calcitriol (1,25-dihydroxyvitamin D3). Calcitriol has a decisive role in the intestinal 
absorption of $\mathrm{Ca}++$; in absence of calcitriol the mineralization of osteoid tissue is diminished and produces rachitis (childhood) and osteomalacia (elderly). Definitive vitamin D deficiency (10-12 $\mathrm{mg} / \mathrm{ml} 25-\mathrm{OH}-\mathrm{D}$ levels) rickets can be prevented or cured by daily supplementation of 200-400 IU of VD (ergocalciferol or cholecalciferol) or 20 000-50 000 IU per month. 1,25-DHCC can also be administered but this therapy needs controlled medical supervision. Calcitriol also controls (together with PTH) expression of RANKL (receptor activator of nuclear factor $\mathrm{kB}$ ), which is important in osteoclast differentiation further some paracrine regulators. In the bones 1,25-DHCC stimulates resorption of calcium. Without vitamin D, only $10-15 \%$ of dietary calcium and ca. $60 \%$ of phosphorus are absorbed [9]. In the parathyroid gland VD-receptors block parathyroid hormone gene expression thus controls proliferation of parathyroid cells. In the kidney, calcitriol weakly stimulates Ca++-reabsorption into the blood.

\section{Hypovitaminosis D in the Elderly}

Table 1: The five main conditions initializing hypovitaminosis D in the elderly.

\begin{tabular}{|c|c|}
\hline Reasons & Explanation \\
\hline Malabsorption & $\begin{array}{l}\text { Gastrectomy, intestinal bypass or resection, inflammatory bowel disease, Crohn's disease, celiac disease, } \\
\text { steatorrhea caused by biliary blockage, pancreatic disease. }\end{array}$ \\
\hline Lack of sunshine & $\begin{array}{l}\text { Living in latitude greater than } 40 \mathrm{o} \text {, north or south, skin covered by cloths (eg. Muslim women), sunscreens } \\
\text { (fear of melanoma), high grade of industrial pollution, household and institutionalized living conditions. }\end{array}$ \\
\hline Chronic kidney dis. & $\begin{array}{l}\text { Healthy kidney function is needed for the } 1 \text { a-hydroxylation reaction, otherwise hyperparathyroidism and } \\
\text { renal bone disease may develop. }\end{array}$ \\
\hline Parathyroid Hormone (PTH) Deficiency & Calcitriol production is diminished. \\
\hline Calcitonin Over prod. & $1,25-(\mathrm{OH}) 2 \mathrm{VD}$ production in the kidney inhibited. \\
\hline
\end{tabular}

It should be mentioned that, as known, kidney function - due to decreased renal blood flow - declines by aging, ca. $10 \%$ per decades after 40 years of age. Due to this fact the final activation of precursors of 1,25-(OH)2-D declines by ageing. In severe chronic kidney disease administration of the active 1,25-DHCC is recommended.

However, line of studies demonstrate that HypoVD is causative of several non-skeletal chronic disease as well and worsens mor-
In the very old population, ( $>80$ y.o.) prevalence of hypovitaminosis D (HypoVD) is much higher than that in the younger counterparts moreover, according to a recent European study of 217 consecutively geriatric hospitalized patients, despite vitamin D supplementation, $44 \%$ of them remained VD deficient [10]. In hospitalized very old patients, a higher comorbidity burden is associated with HypoVD, moreover this is a predictor of higher length of hospital stay [11,12]. Most cases of hypovitaminosis D is asymptomatic therefore only a portion of patients with HypoVD gets medical support. Measurement of the muscular functions in the lower extremities may indicate insufficient vitamin D levels. Low VD blood levels are usually accompanied with low calcium and phosphate concentrations, while alkaline phosphatase and PTH levels are elevated. Serious sequel of vitamin D deficiency is hypocalcemia with subsequent convulsions and in the last resort tetany, again, in long-term VD-insufficiency osteomalacia develops. Low vitamin D levels may occur as result of five frequently realized reasons demonstrated in Table 1.

Table 2: The main clinical consequences of hypovitaminosis D and/or vitamin D supplementation in the elderly.

\section{Consequence}

\section{Deterioration in} bone health

Deterioration of skeletal muscle system

\begin{tabular}{|c|c|}
\hline \multicolumn{2}{|r|}{ 2. Extra skeletal consequences } \\
\hline Cancer prevention? & controversial results in initiation however positive results in prevention (breast, colon). \\
\hline $\begin{array}{l}\text { Cardiovascular pre- } \\
\quad \text { vention? }\end{array}$ & $\begin{array}{c}\text { cardiomyopathy and congestive heart failure in children due to HypoVD are reversible with VD supplementation; strokes, } \\
\text { atherosclerosis, coronary artery disease show relationship with HypoVD; inverse association between HypoVD and high blood } \\
\text { pressure however RCTs could not confirm the observations. }\end{array}$ \\
\hline T2DM & $\begin{array}{l}\text { A large-scale meta-analysis highlighted the inverse correlation between developing T2DM and VD status. However, no evidence } \\
\text { exists that VD supplementation could reduce Type-II diabetes mellitus. }\end{array}$ \\
\hline Psychiatric disorders & $\begin{array}{c}\text { Cognitive impairment of elderly patients is associated with substantial cognitive decline. High dose VD treatment significantly } \\
\text { improve depressive symptoms after } 1 \text { year }\end{array}$ \\
\hline Skin diseases & $\begin{array}{l}\text { Psoriasis is one of the few non skeletal diseases where the correlation with HypoVD has been demonstrated and the actions of } \\
\text { VD have well-validated clinical application. }\end{array}$ \\
\hline liver disease & $\begin{array}{l}\text { Low vitamin D levels were inversely associated with the histological severity of non-alcoholic liver disease and non-alcoholic } \\
\text { steatohepatitis. }\end{array}$ \\
\hline immunity & $\begin{array}{c}\text { in adaptive immunity, association studies found inverse correlation between VD and/or VD intake and a number of autoim- } \\
\text { mune diseases. }\end{array}$ \\
\hline
\end{tabular}

1. Skeletal consequences

Diminished $\mathrm{Ca}++$ (and $\mathrm{Mg}_{++}, \mathrm{PO}_{4}{ }^{3}$ ) absorption from gut and subsequent diminished bone mineralization, in the elderly osteomalacia. Dietary support with VD is recommended in the elderly, in combination with calcium.

Falls in VD deficient elderly women correlates with the HypoVD and supplementation with 700-1000 IU/d significantly improved risk of falls. bidity prevalence in the aged ( $>50$ y.o). A pleiotropic effect appears in the extra-skeletal systems: the cardiovascular system, the nervous system, the immune system and the cancerous tissues are affected alike (Table 2). Though in vitro and in vivo (mainly animal) studies demonstrate the disease-inducing effect of HypoVD, human RCTs usually fail to prove preventive or therapeutic beneficial consequence of vitamin D supplementation. 
Beyond the conditions inserted in Table 1. two other factors in connection with pharmacotherapy should be negotiated: the polypharmacy and the medication adherence. There are pharmacological effects that either as side effect or as interaction may decrease VD levels of individuals. Long-term treatment with anticonvulsants (even as adjuvant in pain-relief) may decrease the activation process of VD in the liver. Phenobarbital and phenytoin increase the hepatic metabolism (inactivation) of vitamin D. Corticosteroids inhibit $\mathrm{Ca}++$ absorption, which results in impaired VD metabolism. Thiazide-type diuretics may induce hypercalcemia and subsequent arrhythmia in patients on digitalis therapy. Co-administration with magnesium-containing medicines/dietary supplements (incl. antacids, too) should be also avoided. Since VD is fat soluble, certain drugs affecting the fat absorption (orlistat, cholestyramine) can reduce the intestinal absorption of vitamin D. In contrast: vitamin D supplementation may decrease the serum levels of statins, eg. atorvastatin due to its inductive effect on CYP3A4 by which atorvastatin is partially metabolised. This effect may influence metabolism of a lot of drugs depending on the activity of hepatic isoenzyme CYP3A4, however the reports on this type of interaction are scarce.

Finally, in this age group one should not forget about the adherence to drug administration. Medication failures are more frequent in the elderly especially who live alone, and the missing administration of drugs and/or dietary supplements are also accidental ground of lack of effect [13].

In the elderly, melanin, which is very effective UVB absorber, often decreased in the skin pigmentation, thus the dermic synthesis of VD is also reduced. The moderate use of modern sunscreens does not inhibit the vitamin D synthesis in the epidermis, in contrast to strict physical avoidance of sunlight $[14,15]$ ! In contrast creams of the older generation (main ingredient: para-aminobenzoic acid) with sun protection factor 30 reduced VD synthesis in the skin by more than 95\%, according to an assessment of 1987 [16]. To avoid increased risk of HypoVD, there have been developed reliable self-administered VD status predictors that can used by older patient as well [17].

Interestingly, Gorter et al. [18] published some years ago that along with the often referred risk factors (elevated age, non-Caucasian skin type, winter, smoking) main protective factors against severe vitamin D deficiency are VD supplementation, higher daily sun exposure and the regular alcohol consumption. The last one, of course, does not cover the uncontrolled drinking habit.

\section{Hypovitaminosis D in the Obese}

Durá-Travé and co-workers published a survey of 564 young (3,6-15,8 yo) Caucasian individuals in Spain and revealed that prevalence of hypovitaminosis D (HypoVD) was extra high $(81,1 \%)$ in severe obese and high $(68,2 \%)$ in obese [19]. In contrast, HypoVD was present in the half of individuals having normal $(58,1 \%)$ or overweight (55\%). Numerous studies with old participants support the inverse association of se-25-OH-D and BMI greater than $30 \mathrm{~kg} /$ $\mathrm{m}^{2}$ thus obesity is irrespective of age associated with VD deficiency, however, the causative role of HypoVD in development of obesity is still not proven. Obese people in general, show also higher serum PTH level than healthy weight people. As known, PTH levels are inversely associated with vitamin D levels and patients with hyperparathyroidism are on average more obese than euparathyroid population [20].

The elevated potency of adipose tissue to store VD has long been known but only recent studies revealed that vitamin D recep- tors and vitamin D metabolizing enzymes are expressed in adipocytes as well. By this presence VD influence the regulation of adipogenic gene expression incl. lipogenesis and lipolysis, cytokine release, NF-kB signaling (low grade inflammation process), etc. has been demonstrated [21]. Thus, the relation between VD status and obesity-beyond the storage of this fat-soluble vitamin is not just a hypothesis anymore.

Vitamin D also affects the appetite regulation through the leptin-production and the carbohydrate metabolism via influencing insulin-sensitizing hormone release. There are many hypotheses about the details of HypoVD link with overweight/obesity. One of them is that low serum 25-OH-vitamin D levels block leptin and increase adiponectin release therefore these adipokine levels facilitate fat deposition. The opposite situation has also been proved: in a study 32 patients received weekly $50000 \mathrm{IU}$ vitamin D and the leptin level moderately increased, the adiponectin level moderately decreased in comparison to 32 control patients of the similar mean age (62 and 60 years, respectively) [22]

Clinical studies in this field are, unfortunately, heterogenous. Analysis of observational studies and randomized controlled trials (RCTs) present controversial picture: observational studies supported the inverse association between leptin and 25-OH-VD but RCTs failed to support this result [23].

More and more obese patients choose the surgical solution. Bariatic surgery does not reverse the vitamin D deficiency of obesity, rather such surgery makes HypoVD worse due to the limited absorption surface.

Thus, the relation between obesity and HypoVD is ambiguous and results off small studies are not supported with high value randomized linical trials. VD supplementation may prevent obesity, but it does not lead to weight loss in obese subjects. It is, however, clear that due to the high distribution potential of VD in the adipose tissue, a large amount of VD disappears from the circulation therefore supplementation with higher doses is necessary if VD deficiency is verified.

\section{The D-Hypervitaminosis}

Due to an inborn defence mechanism if active VD (1,25-dihydroxycholecalciferol) is sufficient, the molecule is further metabolized by CYP24 enzymes in the liver and the kidney (Figure 3). These metabolites are already inactive. Besides and among other regulatory factors, the activation step 25-OHD3 to 1,25(OH)2D3 is age dependent, ie. in the elderly production rate of calcitriol is diminished [24].

Even so, rarely and mostly in the early childhood, D-hypervitaminosis may occur. This is usually due to inborn error the hydroxylation of cholecalciferol and it results in extreme high se-levels of 25-OH-vitamin D. In the adults, this kind of toxicosis may not come true because even in high 25-OH-VD levels 1,25-dihydroxy-VD is relatively low. The regulator is the hydroxylation process in the kidney and the difference of intracellular receptor-affinity: VD-nuclear receptor affinity to calcitriol is 1000 -fold of $25-0 H-V D$. Moreover, the affinity of plasma transport-proteins is ca. 200x higher of 25-0HVD than that of calcitriol thus overdosing of VD can hardly reach as high concentration that would evoke adverse effects. Moreover, according to a study with 38 patients, 10000 IU vitamin D per day for 4 months resulted in no adverse effects, it means vitamin D has a wide therapeutic window [25]. In very rare cases and in very high doses (eg. overdosage of supplements or medical [iatrogenic] inter- 
vention) transport-proteins may be saturated and, in this situation the intracellular receptors may be activated. Some 5 years ago there was published a case report about an infant receiving inadvertently $50.000 \mathrm{IU} /$ day for two months. This resulted in hypercalcemia, hypercalciuria and nephrocalcinosis Wani et al. [26]. One year later reported a malpractice in India: 62 patients got 600,000 IU/im. injection for correction of vitamin D deficiency within some weeks [27]. Prescriptions of mega doses $(1,000,000 \mathrm{IU}$ and above within a short period of time) were reported in 2017 as well [28]. Of course, toxic symptoms incl. acute kidney injury developed in these patients. (Median age of the patients in the last publication was 67,7 years!) Toxicosis, in general, induce toxic symptoms like severe hypercalcemia/hypercalciuria, confusion, apathy, vomiting, abdominal pain, polyuria/anuria, polydipsia, dehydration, azotemia or in chronic toxicity development of kidney stones and/or calcification of organs (kidney, heart). Acute kidney injury can be life-threatening and may associate with morbidity if not identify early enough Usually these phenomena manifest above the serum-level of 150 $\mathrm{ng} / \mathrm{ml}$ however there are reports about hypervitaminosis D without alarming signs even in a serum levels of $195 \mathrm{ng} / \mathrm{ml}$ [29]. Major risk factors of VD toxicity include elevated age, coexisting chronic kidney disease, use of thiazide diuretics, genetic predisposition (eg. CYP24 deficiency), high doses of parenteral administration of the vitamin D. The elevated calcium is an indicator of possible hypervitaminosis D but not definitive marker of elevated VD levels. (Hypercalcemia may occur due to several reasons incl. long-term immobilization, hyperparathyreosis, malignancies [eg. breast, pancreas, uterus carcinoma], sarcoidosis, milk-alkali syndrome, etc.)

After cessation of VD-administration the normalization of se-level take several weeks or months probably due to its deposition in adipose tissue.

\section{CONCLUSION}

Vitamin D is a hormone called vitamin organizing the calcium and bone homeostasis as main job with wide range of extra-skeletal effects. Its role is too complex to treat deficiency via OTC drugs and/ or nutrition supplements based on self-diagnosis. Its treatment value in the skeletal system have been verified and the vitamin D therapy - together with calcium supplementation - became long accepted part of the medicine.

Although myriad of observational studies suggests the correlation between low vitamin D intake and several other diseases, results of promising in vitro and animal studies demonstrating preventive and/or curative effect of VD supplementation could still not be proved by well-designed clinical trials in the course of every extraskeletal disorder. As it has been evidenced that vitamin D receptors can be found in almost all organs and tissues, it alludes to the important role of VD. All above listed facts support the assumption that maybe the complexity of the pathways results in the absence of direct action of VD or VD deficiency in manifestation of certain diseases or in treatment efforts. Most probably this is like a chain, where many links are needed to keep the binding to finally release an effect and vitamin D is just one link among a lot.

Vitamin D deficiency is a relative phenomenon, depending on the definition of threshold. Due to the wide range of risk factors leading to HypoVD, many individuals need correction in vitamin D status. Maybe the non-pharmaceutical options of correction are not really exhausted. The high consumption figures of dietary supplements are, however, supposedly directed by business considerations.
In gastrointestinal disorders, especially in the elderly, when malabsorption of vitamin D and declining kidney functions are present or probable, administration of vitamin D supplementation is recommended. The therapeutic amount should be fitted to the degree of deficit; without laboratory measurement 800-1200 IU per day advisable for elderly above 70 years and for obese individuals above $30 \mathrm{~kg} / \mathrm{m}^{2}$.

\section{REFERENCES}

1. Yu S, Fang H, Han J, Cheng X, Xia L, et al. (2014) The high prevalence of hypovitaminosis D in China. medicine (Baltimore) 94(8): e585.

2. Williams R, Novick M, Lehman E (2014) Prevalence of hypovitaminosis $\mathrm{D}$ and its association with comorbidities of childhood obesity. Perm J 18(4): 32-36.

3. Alzaheb RA (2018) The Prevalence of Hypovitaminosis D and its associated risk factors among women of reproductive age in Saudi Arabia: A systematic review and meta-analysis. Clin Med Insight Womens Health11: 1179562X18767884.

4. Kamboj P, Dwivedi S, Toteja DS (2018) Prevalence of hypovitaminosis D in India \& way forward. Indian J Med Res 148(5): 548-556.

5. Annweiler C, Riou J, Alessandri A, Gicquel D, Henni S, et al. (2017) Clinical Identification of Geriatric Patients with Hypovitaminosis D: The 'Vitamin D Status Predictor for Geriatrics' Study. Nutrients 9(7): 658

6. Caccamo D, Ricca S, Curro M, Ientile R (2018) Health risks of hypovitaminosis D: A review of new molecular insights. Int J Molec Sci 19: 892.

7. (2020) NIH National Institute of Health, Office of dietary supplements: Vitamin D. Fact sheet for health Professionals.

8. Glossmann HH (2010) Origin of 7-dehydrocholesterol (Provitamin D) in the skin. J Investigative Dermatol 130: 2139-2141.

9. Nair R, Maseeh A (2012) Vitamin D: The sunshine vitamin. J Pharmacol Pharmacother 3(2): 118-126.

10. Boettger SF, Angersbach B, Klimek CN, Monteiro Wanderley AL, Shaibekov A, et al. (2018) Prevalence and predictors of vitamin D-deficiency in frail older hospitalized patients. BMC Geriatrics 18: 219.

11. Hélard L, Mateus-Hamdan L, Beauchet O, Annweiler C (2013) Hypovitaminosis D in geriatric acute care unit: a biomarker of longer length of stay. Disease Markers 35(5): 525-529.

12. Boccardi V, Lapenna M, Gaggi L, Garaffa FM, Croce MF, et al. (2019) Hypovitaminosis D: a disease marker in hospitalized very old persons at risk of malnutrition. Nutrients 11(1): 128.

13. Mahmoodi H, Nahand FJ, Shaghaghi A, Shooshtaghi S, Jafarabadi MA, et al. (2019) Gender based cognitive determinants of medication adherence in older adults with chronic conditions. Patient Preference Adherence 13: 1733-1744.

14. Sample A, He YY (2018) Mechanism and prevention of UV-induced melanoma. Photodermatol Photoimmunol Photomed 34(1): 13-24.

15. Pereira LA, Luz FB, Moraes de Oliveira, Carneiro CM, Rampazzo XAL, et al. (2019) Evaluation of vitamin D plasma levels after mild exposure to the sun with photoprotection. A Bras Dermatol 94(1): 56-61.

16. Matsuoka LY, Ide L, Wortsman J, MacLaughlinb JA, Holick MF (1987) Sunscreens suppress cutaneousnvitamin D3 synthesis. J Clin.Endocrinol Metab 64(6): 1165-1168.

17. Annweiler C, Kabeshova A, Callens A, Paty ML, Duval GT, et al. (2017) Self-administered vitamin D status predictor: older adults can use a selfquestionnaire for evaluating their vitamin D status. PLoS ONE 12(11): e0186578.

18. Gorter EA, Krijnen P, Schipper IB (2016) Vitamin D deficiency in adult fracture patients: prtevalence and risk factors. Eur J Trauma Emerg Surg 42: 369-378. 
19. Durá-Travé T, Galinas-Victoriano F, Chueca-Guindulain MJ, BerradeZubiri S (2017) Prevalence of hypovitaminosis D and associated factors in obese Spanish children. Nutr Diabet 7: e248.

20. Tran H, Grange JS, Adams-Huet B, Nwariaku FE, Rabaglia JL, et al. (2014) The impact of obesity on the presentation of primary hyperparathyroidism. J Clin Endocrinol Metab 99(7): 2359-2364.

21. Abbas MA (2017) Physiological functions of vitamin D in adipose tissue. J Steroid Biochem Mol Biol 165(Pt B): 369-381.

22. Naini AE, Vahdat S, Hedaiati ZP, Shahzeidi S, PezeshkiAH, et al. (2016) The effect of vitamin D administration on serum leptin and adiponectin levels in end-stage renal disease patients on hemodialysis with vitamin D deficiency: a placebo-controlled double-blind clinical trial. J Res med Sci 21: 1 .

23. Hajimohammadi M, Shab-Bidar S, Neyestrani TR (2017) Vitamin D a serum leptin: a systematic review and meta-analysis of observational studies and randomized clinical trials. Eur J Clin Nutr 71(10): 11441153

24. Anderson PH, May BK, Morris HA (2003) Vitamin D metabolism: New concepts and clinical implications. Clin Biochem Rev 24: 13-26.
25. Amir E, Simmons CE, Greedman OC, Dranitsaris G, Cole DEC, et al. (2010) A phase 2 trial exploring the effects of high-dose (10,000 IU/ day) vitamin D3 in breast cancer patients with bone metastases. Cancer 116: 284-291.

26. Ketha H, Wadams H, Lteif A, Singh RJ (2015) Iatrogenic vitamin D toxicity in an infant- A case report and review of literature. J Steroid Biochem Mol Biol 148: 14-18.

27. Wani M, Wani I, Banday K, Ashraf M (2016) The other side of vitamin D therapy: A case series of acute kidney injury due to malpractice-related vitamin D intoxication. Clin Nephrol 86(11): 236-241.

28. Chowdry AM, Azal H, Najar MS, Mir I (2017) Acute kidney injury due to povercorrection of hypovitaminosis D: a tertiary center experience in the Kashmir Valley of India. Saudi J Kidney Dis Transpl 28(6): 13211329.

29. Rahesh J, Chu V, Peiris AN (2020) Hypervitaminosis D without toxicity. Proc (Bayl Univ Med Center) 33(1): 42-43. 